University of Nebraska - Lincoln

DigitalCommons@University of Nebraska - Lincoln

November 2003

\title{
Effects of neurobehavioral assessment on feeding and weight gain in preterm neonates
}

T. E. Senn

K. A. Espy

University of Nebraska-Lincoln, kespy2@unl.edu

Follow this and additional works at: https://digitalcommons.unl.edu/dcnlfacpub

Part of the Neurosciences Commons

Senn, T. E. and Espy, K. A., "Effects of neurobehavioral assessment on feeding and weight gain in preterm neonates" (2003). Developmental Cognitive Neuroscience Laboratory - Faculty and Staff Publications. 14. https://digitalcommons.unl.edu/dcnlfacpub/14

This Article is brought to you for free and open access by the Developmental Cognitive Neuroscience Laboratory at DigitalCommons@University of Nebraska - Lincoln. It has been accepted for inclusion in Developmental Cognitive Neuroscience Laboratory - Faculty and Staff Publications by an authorized administrator of DigitalCommons@University of Nebraska - Lincoln. 


\title{
Original Articles
}

\section{Effects of Neurobehavioral Assessment on Feeding and Weight Gain in Preterm Neonates}

\author{
THERESA E. SENN, M.A. \\ Department of Psychology, Southern Illinois University, Carbondale \\ KIMBERLY ANDREWS ESPY, PH.D. \\ Department of Family and Community Medicine, Southern Illinois University School of Medicine, Carbondale, Illinois
}

\begin{abstract}
Neonatal intensive care unit personnel and parents often are concerned that developmental assessment will tire preterm neonates and impair their feeding ability and subsequent weight gain. Therefore, the amount of fluid consumed by 108 preterm neonates ( $\leq 36.5$ wk gestational age) was compared before and after administration of the Neurobehavioral Assessment of the Preterm Infant (NAPI). In addition, the weight gain of 35 preterm neonates who were administered the NAPI was compared with that of a matched control group of 35 preterm neonates who were not administered the NAPI. There were no differences in the amount of fluid consumed before and after NAPI administration, and there were no differences in weight gain between neonates who were and were not administered the NAPI. The effects of NAPI administration did not differ as a function of gestational age group. Therefore, the NAPI can be administered safely to preterm neonates without affecting feeding performance or weight gain. J Dev Behav Pediatr 24:85-88, 2003. Index terms: neurobehavioral assessment, preterm infant, feeding, weight gain.
\end{abstract}

The Neurobehavioral Assessment of the Preterm Infant $(\mathrm{NAPI})^{1}$ is a developmental assessment specifically designed for use with preterm neonates. NAPI administration involves observing the neonate's behavioral states, gently moving the neonate's arms and legs to test strength and reflexes, and shaking a rattle and talking to the neonate to see how well the neonate orients to, and tracks, visual and auditory stimuli. ${ }^{1}$ It is slow-paced and gentle and does not include more aversive items sometimes found in other newborn assessments (e.g., pin prick). Therefore, NAPI administration should not adversely affect the neonate. Clinical experience in the neonatal intensive care unit (NICU), however, suggests that parents and health care personnel often are reluctant to permit NAPI administration, particularly in preterm neonates born earlier in gestation. Nurses have expressed concerns that NAPI administration before a scheduled nipple feeding (feeding either from a bottle or directly from the breast) may tire the neonate and negatively impact his or her subsequent fluid consumption. As a result, parents often are concerned that NAPI administration will influence the neonate's weight gain negatively, thus requiring a longer hospital stay.

Received May 2, 2002; accepted October 23, 2002.

Address for reprints: Theresa E. Senn, M.A., Department of Psychology, Southern Illinois University, Mailcode 6502, Carbondale, IL 62901-6502; e-mail: teri@siu.edu.
Indeed, certain types of stimulation may be harmful to neonates. For example, researchers found that neonates responded positively to someone talking in "motherese," but that they responded with negative faces and less time spent with eyes open when talking was combined with touch. ${ }^{2}$ Certain types of touch, such as a heel stick, are known to produce negative reactions, such as crying and grimacing, and to result in greatly reduced oxygenation levels. ${ }^{3}$ It is possible that NAPI administration, with the various types of stimulation provided, may overstimulate preterm neonates and negatively affect feeding and weight gain.

Although the NAPI is not an intervention tool, data from other studies on the effects of neonatal stimulation indicate that the handling that occurs during NAPI administration may not be harmful to neonates. For example, massage has been associated with better performance on a habituation task in 4-month-old infants. ${ }^{4}$ In preterm neonates, massage resulted in more time spent in active and alert states, less time in the hospital, and more mature performance on the Brazelton Neonatal Behavioral Assessment Scale. ${ }^{5,6}$ Massage may even have long-term cognitive benefits; for example, Field and colleagues ${ }^{5}$ found that preterm neonates who were massaged during hospitalization scored higher on the Bayley Scales of Infant Development ${ }^{7}$ at 6 months of age.

Furthermore, touch stimulation has been shown to positively affect weight gain in preterm neonates. Preterm neonates who were assigned randomly to be massaged gained more weight during hospitalization, despite the fact that calorie consumption did not differ among groups. ${ }^{5}$ 
Touch, by skin to skin contact, is also a critical component of kangaroo care, a program that has been associated with greater weight gain in preterm neonates. ${ }^{8}$

Several mechanisms for the beneficial effects of interventions that include touch on weight gain have been proposed, including reduced production of stress hormones (e.g., cortisol) and catecholamines, ${ }^{9}$ and increased vagal activity that leads to higher levels of insulin, a digestive hormone. ${ }^{10}$ In one study, preterm neonates who received tactile stimulation had a greater reduction in gastric $\mathrm{pH}$ than did a control group, leading the researchers to conclude that touch promoted weight gain by aiding digestion. ${ }^{11}$ Therefore, the touch and stimulation that is a part of NAPI administration may not negatively affect preterm neonates.

Given the conflicting evidence about the effects of different types of stimulation on preterm neonates, it is important to determine whether or not NAPI administration negatively affects neonatal feeding and growth. However, few researchers have investigated the effects of NAPI administration on preterm neonates. Morrow et $\mathrm{al}^{3}$ demonstrated only slight differences in oxygenation levels during NAPI administration, although the effects on neonate feeding and weight gain were not investigated. Given our clinical experience in the NICU, we investigated (1) the effects of NAPI administration on neonatal feeding and (2) the effects of NAPI administration on weight gain during the period of NAPI administration. Specifically, NAPI administration was not expected to affect feeding or weight gain.

\section{STUDY I METHODS}

The first study aim was investigated by comparing fluid (breast milk or formula) consumption before and after administration of the Neurobehavioral Assessment of the Preterm Infant (NAPI) in 108 preterm neonates (64 female [59\%], 44 male [41\%], 89 white [82\%], and 19 minority $[18 \%]$; gestational age $\leq 36.5 \mathrm{wk})$ who participated from October 1997 to July 2000 as part of a larger study of preterm infant development (Table 1). All neonates were hospitalized in an advanced care Level II neonatal intensive care unit (NICU) (with an exception to treat neonates as young as 26 wk gestational age who do not require surgery) of a regional, rural hospital. Neonates with any significant medical complications (e.g., bronchopulmonary dysplasia, seizure disorder, and Grade III or Grade IV intraventricular

Table 1. Sample Characteristics of Study I Participants

\begin{tabular}{lcc}
\hline & Mean & SD \\
\hline Infant gestational age, wk & 32.4 & 2.3 \\
Infant birth weight, g & 1782 & 422 \\
Infant age at discharge, d & 25.5 & 16.8 \\
Percentage of days received breast milk & 17.7 & 24.7 \\
Total days on oxygen & 3.3 & 7.0 \\
Total days on ventilation & 0.6 & 1.3 \\
Maternal age, yr & 24.9 & 6.6 \\
Number of NAPI evaluations & 4.9 & 2.8
\end{tabular}

NAPI, Neurobehavioral Assessment of the Preterm Infant. Not all data available in all infant charts; numbers range from 104 to 108 per cell. hemorrhage) were excluded from participation, because the effect of administration might be obscured in neonates with such severe sequelae.

Once the neonate was medically stable (i.e., the neonate had no trailing intravenous line, the neonate was not receiving oxygen therapy, and the nurse considered the neonate well enough to be assessed), the NAPI was administered by trained examiners every other day during the remainder of the neonate's hospital stay to examine neurobehavioral change during a period of greatest behavioral instability. This schedule was chosen because the aims of the larger study were to examine the neurobehavioral developmental trajectories during the neonatal period in which there is considerable behavioral instability, necessitating frequent observations. ${ }^{12}$ Occasionally, the NAPI was not administered on a particular day because of medical condition or scheduled procedures. NAPI administration began approximately 45 to 60 minutes before the next scheduled feeding, and the assessment took approximately 30 minutes to complete. On average, each neonate was assessed 4.9 times $(\mathrm{SD}=2.8)$ during hospitalization. After discharge, the neonates' medical charts were reviewed, and information on medical condition, fluid consumption, and weight gain was collected.

To determine whether NAPI administration had an effect on neonate feeding, the amounts of formula or breast milk consumed by the neonate before NAPI administration, immediately after NAPI administration, and at the second feeding after NAPI administration were obtained from the neonate's medical chart. Neonates were fed a prescribed amount on a fixed schedule based on medical status, but neonates did not always consume the given amount. Only the first NAPI administered after the neonate was on full nipple feeding was examined in the present study. Only NAPI administration after full nipple feeding was considered, because it was not expected that the evaluation would affect the amount of milk consumed through gavage feedings (the amount delivered during a gavage feeding is not dependent on the neonate). This NAPI administration occurred, on average, when the neonate was 20 days old $(\mathrm{SD}=16)$. Excluded from the sample were neonates who fed directly from the breast after the relevant NAPI administration, because the amount of breast milk consumed could not be quantified. Repeated measures analyses of variance (ANOVA) were used to determine whether amount of fluid consumed differed before and after NAPI administration.

\section{STUDY I RESULTS}

Administration of the Neurobehavioral Assessment of the Preterm Infant (NAPI) did not affect the amount of fluid (formula or breast milk) consumed by the neonates. The amount of fluid consumed at the feeding before NAPI administration and the amount consumed immediately after NAPI administration did not differ, $\mathrm{F}(1,107)=.48, p>.48$. Before NAPI administration, neonates consumed an average of $37.5 \mathrm{~mL}$ of fluid $(\mathrm{SD}=6.5$ ), whereas neonates consumed an average of $37.7 \mathrm{~mL}$ of fluid $(\mathrm{SD}=6.4)$ immediately after NAPI administration. There also was no evidence of a delayed effect of NAPI administration on feeding, because 
there were no differences between the amount consumed before the evaluation and the amount consumed at the second feeding after evaluation, $\mathrm{F}(1,106)=1.57, p>.21$. On average, neonates consumed $37.8 \mathrm{~mL}$ of fluid $(\mathrm{SD}=6.5)$ at the second feeding after NAPI administration.

It is possible that NAPI administration more easily tires neonates who are born at earlier gestational ages or who are of a younger conceptional age (gestational age plus chronological age since birth) at the time of evaluation, and therefore NAPI administration would adversely affect feeding performance to a greater degree in these neonates. The effect of gestational age on fluid consumption was examined using a repeated measures analysis of variance (ANOVA), with the amount of fluid consumed before and after NAPI administration as the within-subjects factor and gestational age category ( $\leq 32 \mathrm{wk} ; \mathrm{n}=47 \mathrm{vs}>32 \mathrm{wk} ; \mathrm{n}=$ $61)$ as the between-subjects factor. The amount of fluid consumed before and after NAPI administration did not differ as a function of gestational age group for the first feeding after evaluation, $\mathrm{F}(1,106)=.53, p>.46$, or for the second feeding after evaluation, $\mathrm{F}(1,105)=.05, p>.82$. Similarly, neonates of younger conceptional age $(\leq 35 \mathrm{wk}$; $\mathrm{n}=44)$ and older conceptional age $(>35 \mathrm{wk} ; \mathrm{n}=64)$ did not differ in fluid consumption before and after NAPI administrations, $\mathrm{F}(1,106)=.26, p>.60$, or in the amount of fluid consumed before evaluation and at the second feeding after evaluation, $\mathrm{F}(1,105)=0, p>.97$.

\section{STUDY II METHODS}

Because parents and nurses often are concerned that administration of the Neurobehavioral Assessment of the Preterm Infant (NAPI) will deleteriously affect the neonate's weight gain, it was important to determine whether NAPI administration affected weight gain over the course of NAPI administrations. Therefore, to investigate the second study aim, a comparison control group of neonates born in the hospital from January 1997 to December 1998 who did not participate in the study on development was obtained (No NAPI group). The medical charts of all preterm neonates born during this period were reviewed. The No NAPI group neonate participants were matched on sex, race, and birth gestational age to neonates who had received at least one NAPI administration (NAPI group). If more than one match was available, the No NAPI neonate closest in birth weight to the target NAPI group neonate was used.

In the NAPI group, the neonate's age at first NAPI administration was calculated. The neonate's average daily weight gain from first NAPI administration to hospital discharge was calculated by subtracting the neonate's weight on the day the NAPI was first administered from the discharge weight and then dividing by the number of days the neonate was hospitalized between first NAPI administration and discharge. To be included in the NAPI group, neonates had to be in the hospital at least 5 days after the first NAPI administration so that weight gain during the period of NAPI administration could be assessed adequately. To determine average daily weight gain in the No NAPI group, a similar procedure was used. Weight for each No NAPI group neonate on the same day
Table 2. Sample Characteristics of Study II Participants

\begin{tabular}{lccccc}
\hline & \multicolumn{2}{c}{ NAPI } & & \multicolumn{2}{c}{ No NAPI } \\
\cline { 2 - 3 } Variable & Mean & SD & & Mean & SD \\
\hline Infant gestational age, wk & 32.4 & 2.1 & & 32.4 & 2.1 \\
Infant birth weight, g & 1735 & 371 & & 1838 & 401 \\
Infant age at discharge, d & 24.7 & 14.8 & & 26.9 & 14.0 \\
Weight gain, g & 395 & 328 & & 425 & 375 \\
Percentage of days received & 21.6 & 29.7 & & 26.4 & 29.7 \\
$\quad$ breast milk & & & & \\
Total days on oxygen & 1.6 & 3.5 & & 2.8 & 3.4 \\
Total days on ventilation & 0.6 & 1.2 & & 1.6 & 1.8 \\
Maternal age, yr & 24.4 & 6.2 & & 25.4 & 5.8 \\
\hline
\end{tabular}

NAPI, Neurobehavioral Assessment of the Preterm Infant. Not all data available in all infant charts; numbers range from 25 to 35 per cell.

${ }^{*} p<.05$.

of life that the NAPI was administered to the matched NAPI group neonate was used as the initial weight. Then this value was subtracted from the discharge weight and divided by the number of days remaining in the neonate's hospital stay. Using this procedure, 35 neonates who had at least one NAPI assessment were matched to those who were not assessed.

Characteristics of neonates in the NAPI and No NAPI groups are presented in Table 2. Outliers in number of days on oxygen or number of days of ventilation were replaced with the value equal to $3 \mathrm{SD}$ from the mean. In each group there were 19 male $(54 \%)$ and 16 female neonates $(46 \%)$; $32(91 \%)$ were white, and three $(9 \%)$ were of minority ethnicity. There were no differences between the two groups in sex, race, gestational age, birth weight, age at discharge, weight gained over hospitalization, percentage of days on which they received breast milk, days on oxygen, or maternal age. These groups differed in the average number of days spent on ventilation, $t(63)=2.49, p<.05$, with neonates in the NAPI group requiring an average of 0.6 days of ventilation and neonates in the No NAPI group requiring an average of 1.6 days of ventilation. Therefore, analysis of covariance (ANCOVA), with the number of days on ventilation as a covariate, was used to compare weight gain between the NAPI and No NAPI groups.

\section{STUDY II RESULTS}

Weight gain did not differ between the Neurobehavioral Assessment of the Preterm Infant (NAPI) and No NAPI groups, $\mathrm{F}(1,62)=1.67, p>.20$, regardless of whether covariates were included. After adjusting for the covariates, the NAPI group gained an average of $25.4 \mathrm{~g}$, and the No NAPI group gained an average of $21.9 \mathrm{~g}$ per day after the first NAPI administration.

To investigate whether NAPI administration differentially affected the weight gain of earlier-born preterm neonates, an analysis of covariance (ANCOVA) was performed with weight gain as the dependent variable, group (NAPI vs No NAPI) and gestational age ( $\leq 32 \mathrm{wk}$; $\mathrm{n}=28 \mathrm{vs}>32 \mathrm{wk} ; \mathrm{n}=42$ ) as the independent variables, and days on ventilation as the covariate. The effect of NAPI administration did not differ as a function of gestational age 
group, $\mathrm{F}(1,60)=.10, p>.75$, indicating that the weight gain of neonates born earlier in gestation was not affected by NAPI administration differentially.

\section{DISCUSSION}

Administration of the Neurobehavioral Assessment of the Preterm Infant (NAPI) did not affect the amount of breast milk or formula consumed by preterm neonates who were receiving full nipple feedings. NAPI administration also did not impair weight gain in this sample of preterm neonates. Therefore, parents and health care personnel need not be concerned that NAPI administration deleteriously affects the neonate's ability to nipple feed. In addition, NAPI administration did not differentially affect the feeding performance or weight gain of neonates of earlier gestational age. Although NAPI administration did not impair weight gain, weight gain depends on many variables, including caloric intake, that were not assessed in this study.
The NAPI, perhaps because it was designed specifically to be administered to preterm neonates without adverse items often included in other neonatal assessments, did not impair feeding or weight gain, even when administered repeatedly during the neonate's hospitalization. Although NAPI administration takes approximately 30 minutes, much of this time is spent observing the behavioral state, so the amount of time during which the neonate is stimulated is considerably less than the administration time. On average, the NAPI was administered only once every 6.6 days while the neonate was hospitalized, which may be too infrequent to lead to deleterious effects.

These findings suggest that early neurobehavioral examination, such as the NAPI, does not impair feeding performance or weight gain in preterm neonates. This assessment therefore can be used safely to examine early developmental progress, at least in medically stable preterm neonates hospitalized in a Level II neonatal intensive care unit (NICU).

\section{REFERENCES}

1. Korner AF, Thom VA. Neurobehavioral Assessment of the Preterm Infant Manual. San Antonio, TX: Harcourt Brace Jovanovich; 1990.

2. Eckerman CO, Oehler JM, Medvin MB, Hannan TE. Premature newborns as social partners before term age. Infant Behav Dev. 1994;17:55-70.

3. Morrow CJ, Field TM, Scafidi FA, et al. Transcutaneous oxygen tension in preterm neonates during neonatal behavioral assessments and heelsticks. J Dev Behav Pediatr. 1990;11:312-317.

4. Cigales M, Field T, Lundy B, Cuadra A, Hart S. Massage enhances recovery from habituation in normal infants. Infant Behav Dev. 1997;20:29-34.

5. Field T, Scafidi F, Schanberg S. Massage of preterm newborns to improve growth and development. Pediatr Nurs. 1987;13:385-387.

6. Brazelton TB: Neonatal Behavioral Assessment Scale. 2nd ed. Clinics in Developmental Medicine No. 88. Philadelphia, PA: Lippincott; 1984.
7. Bayley N. Bayley Scales of Infant Development. 2nd ed. San Antonio, TX: The Psychological Corporation; 1993.

8. Anderson GC. Touch and the kangaroo care method. In: Field TM, ed. Touch in Early Development. Ch 4. Mahwah, NJ: Lawrence Erlbaum Associates; 1995:35-51.

9. Field T, Grizzle N, Scafidi F, Abrams S, Richardson S. Massage therapy for infants of depressed mothers. Infant Behav Dev. 1996;19: $107-112$.

10. Field TM. Touch therapy effects on development. Int J Behav Dev. 1998:22:779-797.

11. de Roiste A, Bushnell IRW. The immediate effects of a tactile stimulation programme on premature infants. J Reprod Infant Psychol. 1995; 13:57-62.

12. Espy KA, Senn TE, Cunningham D. Prenatal tobacco exposure: effects on early neonatal development. Presented at the biennial meeting of the Society for Research in Child Development; April 19-22, 2001; Minneapolis, MN.

Announcing the Call for Presentations for

\section{"PROMOTING POSITIVE DEVELOPMENT IN YOUNG CHILDREN: DESIGNING STRATEGIES THAT WORK" \\ Head Start's $7^{\text {th }}$ National Research Conference}

presented by the Administration on Children, Youth and Families,

U.S. Department of Health and Human Services, in collaboration with Xtria, LLC; Columbia University's Mailman

School of Public Health, and Society for Research in Child Development

to be held June 28-July 1, 2004, in Washington, DC.

The Call is available at

http://www.headstartresearchconf.net.

Proposals are due on June 27, 2003.

For more information, please contact Bethany Chirico; hsrc@xtria.com; 703-821-3090 ext. 261. 